Especial: Humanismo e investigación: una actividad permanente en la Escuela de Estudios Generales

\title{
II Parte
}

\section{Las profesoras y profesores de la Escuela de Estudios Generales investigan}

\author{
Importancia Histórica y Ecológica del \\ Parque Nacional Diriá
}

\author{
Ana Lorena Chavarría \\ Universidad de Costa Rica, Costa Rica \\ anachav08@gmail.com \\ https://orcid.org/0000-0001-5383-1930
}

Recibido: 15 de enero de 2019

Aceptado: 4 de febrero de 2019

Resumen: El artículo explora parte de los alcances del Trabajo Comunal Universitario en el Parque Nacional Diriá y comunidadades aledañas. Dicho TCU ha abierto un abanico de posibilidades realmente vitales, dinámicas y efectivas pues, a la vez que conservan, también generan un espacio para la reflexión y el estudio académico y sistemático interinstitucional. Ahí el Trabajo Comunal Universitario (TCU) representa una forma activa de la relación Universidad Sociedad, en la que los estudiantes experimentan un acercamiento no solo al Parque Nacional Diría en sí, su problemática y riqueza natural, sino también una relación directa con las comunidades La Esperanza, Colas de Gallo y Vista del Mar, entre otras.

La acción social desarrollada por la Universidad de Costa Rica en las comunidades antes mencionadas y en el Parque Nacional Diría por parte del TCU, ha sido fundamental no solo para mejorar los cambios de conducta y conocimiento con respecto a las áreas protegidas referentes a su protección, valoración y cuido por parte de las comunidades sino también que las actividades y desarrollo del proyecto contribuya a mejorar la calidad de vida de los habitantes de las comunidades.

Palabras clave: Áreas protegidas de Costa Rica; Trabajo Comunal Universitario; Parque Nacional Diría; Educación ambiental; Universidad - Sociedad

\section{Historical and Ecological Importance of Diriá National Park}

\footnotetext{
(c) (1)(2)

La Revista Estudios es editada por la Universidad de Costa Rica y se distribuye bajo una Licencia Creative Commons Atribución-NoComercial-Compartirlgual 3.0 Costa Rica. Para más información envíe un mensaje a revistaestudios.eeg@ucr.ac.cr.
} 


\section{Especial: Humanismo e investigación: una actividad permanente} en la Escuela de Estudios Generales

The article explores part of the scope of University Community Work in the Diriá National Park and surrounding communities. This TCU has opened a range of possibilities that are truly vital, dynamic and effective because, while they preserve, they also generate a space for reflection and academic and systematic interinstitutional study. There, the University Community Work (TCU) represents an active form of the University - Society relationship, in which students experience an approach not only to the National Park itself, its problems and natural wealth, but also a direct relationship with the La Esperanza, Colas de Gallo and Vista del Mar communities, between others.

The social action developed by the University of Costa Rica in the aforementioned communities and in the National Park Diría by the TCU, has been fundamental not only to improve behavioral changes and knowledge regarding the protected areas related to their protection, valuation and care on the part of the communities but also that the activities and development of the project contribute to improve the quality of life of the inhabitants of the communities.

Keywords: Protected areas of Costa Rica; University Community Work; Diría National Park; Environmental education; University - Society

\section{INTRODUCCIÓN.}

Hay que tener en cuenta que en este momento es crucial para el equilibrio ecológico y la supervivencia de todas las especies de flora y fauna, cualquier política tendente a la conservación de las áreas forestales en general, sobre todo porque lamentablemente cada día son más y más los espacios naturales que sucumben por la tala indiscriminada de los bosques en el planeta Tierra. Cabe señalar y resaltar al respecto que Costa Rica se ha distinguido por llevar a cabo esfuerzos internos materializados y eficaces en materia de conservación y que sin duda alguna, con eso participa de un concierto mundial con muchos otros que se están realizando también a nivel mundial y cuya consigna general es por tanto: la conservación y la promoción de los bosques (Blanco y Mata, 1994, pp113-114); para eso el Estado costarricense impulsa en su legislación, la urgente conservación de la riquezas naturales cuando por ejemplo declara la promoción de una "educación ambiental" como algo de interés público, de carácter obligatorio en

La Revista Estudios es editada por la Universidad de Costa Rica y se distribuye bajo una Licencia Creative Commons Atribución-NoComercial-CompartirIgual 3.0 Costa Rica. Para más información envíe un mensaje a 
Especial: Humanismo e investigación: una actividad permanente en la Escuela de Estudios Generales

todos los ámbitos educativos en todo el país así dicta la Ley Orgánica del Ambiente (№7554 de 1995) y la Ley de Biodiversidad №7788 del año 1998 (Obando, 2002, pp65-66).

En este sentido específico citado, nuestro enfoque fundamentalmente explorará algunos de los alcances y de las características de conservación implicadas en un proyecto nacional claramente dedicado a la ejecución de las iniciativas que si bien, se inspiran en la legislación arriba señalada, sobrepasan a nuestro entender la letra misma de lo que estipulan las leyes abriendo un abanico de múltiples iniciativas realmente vitales, dinámicas y efectivas pues también a la vez que conservan, también abren un espacio para la reflexión y el estudio académico y sistemático inter-institucional como es caso del Parque Nacional Diría.

En cuanto a los razonamientos reales que soportan el esfuerzo local arriba dicho, el investigador Meza (2004) especifica que la relación costo-beneficio entre equilibrio ambiental y bienestar económico y social se ligan con el tipo de actividad que realicen los seres humanos y la posibilidad de que sea sustentable, es decir, que las comunidades aledañas al Parque Nacional Diría, objeto insistimos de nuestro estudio particular, adopten nuevas formas de convivencia y uso de los recursos naturales del área con el fin de intervenir con su conservación.

\section{TÓPICOS IMPLICADOS.}

Es recurrente mencionar en este artículo que, en los años 40 el $80 \%$ del territorio costarricense estaba cubierto de bosque $\left(40.880 \mathrm{~km}^{2}\right)$. También, en una investigación realizada por Universidad de Alberta y el Instituto Tecnológico de Costa Rica en el año 2006 coinciden en resaltar las mismas condiciones exitosas donde se encontraron con una cobertura boscosa que alcanzaba el 51,4\%. Más recientemente, Ruíz (2015) hace referencia que en la década de los 80 cerca del 
Especial: Humanismo e investigación: una actividad permanente

en la Escuela de Estudios Generales

$25 \%$ del territorio costarricense estaba cubierto por bosque, mientras que para el año 2013 el porcentaje de cobertura aumentó a 52\%, es decir que las áreas boscosas se duplicaron en las últimas dos décadas. Nos parece entonces que es de suma importancia una urgente Educación ambiental que comience a informar y a la capacitación de la población costarricense en materia de conservación, así como fomentarla, en particular la ya existente en las áreas protegidas; ello redundará en el beneficio específico de las comunidades y en general de los recursos naturales de nuestro país.

Fig. 1. Muestra el porcentaje de áreas boscosas protegidas por cantón

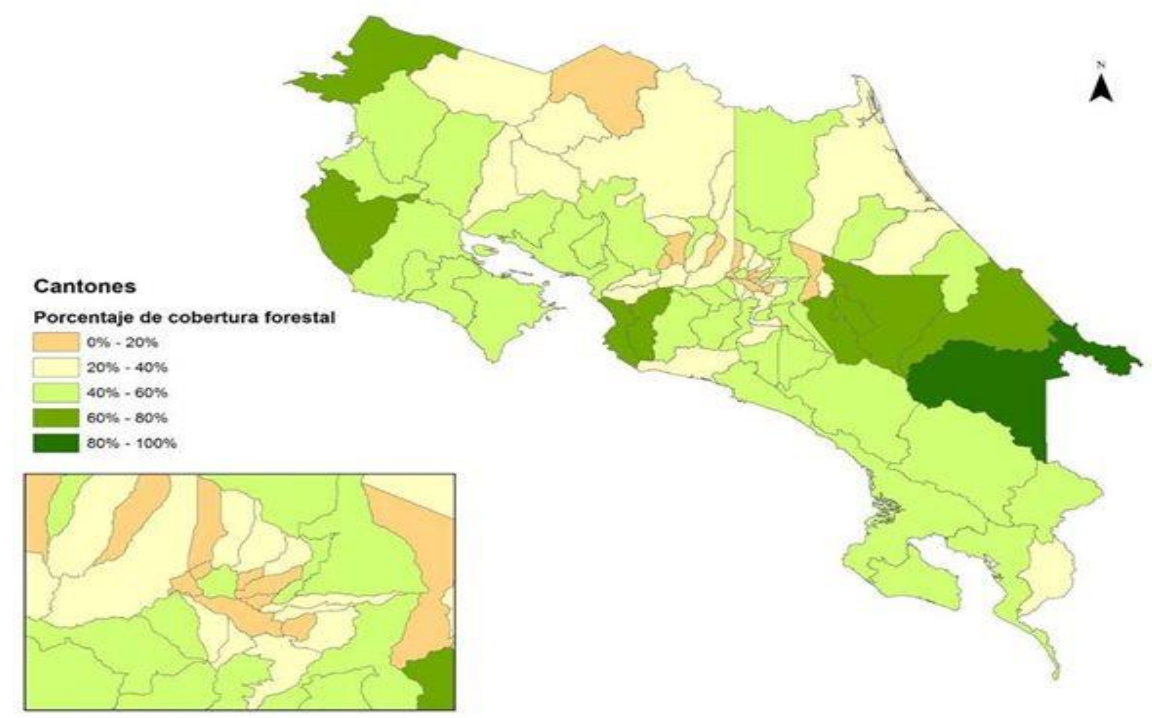

En este mapa, cuanto más oscuro el verde, más porcentaje de área boscosa posee el cantón. (Créditos: Foto Katya Alvarado, Estado de La Nación)

También Costa Rica se ha transformado en uno de los países que ha logrado alcanzar altas tasas de reforestación y tal vez el único en condiciones tropicales que presenta un proceso de recuperación como lo muestran estudios

La Revista Estudios es editada por la Universidad de Costa Rica y se distribuye bajo una Licencia Creative Commons Atribución-NoComercial-CompartirIgual 3.0 Costa Rica. Para más información envíe un mensaje a revistaestudios.eeg@ucr.ac.cr. 
Especial: Humanismo e investigación: una actividad permanente en la Escuela de Estudios Generales

más recientes del Fondo de Financiamiento Forestal de Costa Rica (FONAFIFO 2012). También se pretende la restauración de todo el ecosistema boscoso deteriorado y determinar con exactitud cuál es la calidad de estos ecosistemas. Así mismo se evalúa el comportamiento de la cobertura boscosa utilizando índices biométricos de paisaje y la ecuación de conectividad/ fragmentación, con los cual se identifican los principales cambios en cuanto a distribución y transformación espacial de las categorías de uso de la tierra.

No está demás mencionar de nuevo que en la actualidad las áreas protegidas se encuentran como es lógico bajo presión por las actividades humanas que se expanden conforme la población nacional crece y se desplaza. De igual manera sufren las costas, los ríos y el mar, todos los ecosistemas golpeados además con los cambios climáticos del planeta que se encuentran sometidos a grandes presiones debido a que no se ha encontrado un equilibrio entre el crecimiento económico y la sustentabilidad ambiental.

En efecto, un aspecto relevante que se debe tener en cuenta es sin duda el término de "conservación", la cual implica que debe existir el equilibrio dinámico entre el desarrollo humano y la sostenibilidad de los recursos naturales, en contraste con las políticas económicas. La conservación entonces en nuestros términos, demanda una actitud de respeto a la naturaleza y sus recursos; reconocer su valor y del valor del uso apropiado y previsor para las generaciones futuras que aún no han nacido, ya que debemos dejarles un planeta con suficientes recursos como los que hemos heredado en nuestra generación actual (Padrino, 2010).

Otro concepto importante y recurrente es el de "Educación ambiental", práctica ésta que tiene como objetivo primordial impartir y difundir la conciencia ambiental, el conocimiento ecológico, las actitudes y valores hacia el medio ambiente, facilitándose así tomar compromisos y entablar acciones responsables que tengan como fin último, el uso racional de los recursos lográndose así un

\section{(c) $)(2)(0$}

La Revista Estudios es editada por la Universidad de Costa Rica y se distribuye bajo una Licencia Creative Commons Atribución-NoComercial-CompartirIgual 3.0 Costa Rica. Para más información envíe un mensaje a revistaestudios.eeg@ucr.ac.cr. 
Especial: Humanismo e investigación: una actividad permanente en la Escuela de Estudios Generales

desarrollo adecuado y sustentable. Gonzales Gaudiano (2001) en su artículo "Una nueva lectura a la historia de la educación ambiental" donde cita el principio 19 de la Declaración de Estocolmo de la forma siguiente:

"Es indispensable una labor de educación en cuestiones ambientales, dirigida tanto a las generaciones jóvenes como a los adultos, y que preste la debida atención al sector de la población menos privilegiada, para ensanchar las bases de una opinión pública bien informada y de una conducta de los individuos, de las empresas y de las colectividades, inspirada en el sentido de responsabilidad en cuanto a la protección del medio en toda su dimensión humana. Es también esencial que los medios de comunicación de masas eviten contribuir al deterioro del medio humano y difundan, por el contrario, información de carácter educativo sobre la necesidad de protegerlo, a fin de que el hombre pueda desarrollarse en todos los aspectos" ( pp. 145)

Otra palabra al respecto en materia de Educación ambiental es la que aporta la UNESCO; expresa con los siguientes términos para la consecución educativa:

-Promover la toma de conciencia: concienciar a la gente de los problemas relacionados con el medio y su conservación. La problemática actual respecto a la contaminación y cambio climático ha hecho que el medio ambiente esté en boca de todos y ha aumentado la preocupación de los ciudadanos por las posibles consecuencias que tiene un tratamiento nocivo al medio que nos rodea.

-Conocimientos: ayudar a interesarse por el medio

-Actitudes: adquirir interés por el medio ambiente y voluntad para conservarlo.

-Aptitudes: ayudar a adquirir aptitudes para resolver el problema de la conservación.

-Capacidad de evaluación: evaluar los programas de Educación Ambiental.

-Participación: desarrollar el sentido de responsabilidad para adoptar medidas adecuadas

La Revista Estudios es editada por la Universidad de Costa Rica y se distribuye bajo una Licencia Creative Commons Atribución-NoComercial-CompartirIgual 3.0 Costa Rica. Para más información envíe un mensaje a 
Especial: Humanismo e investigación: una actividad permanente en la Escuela de Estudios Generales

\section{DATOS HISTÓRICOS SOBRE EL PARQUE NACIONAL DIRIÁ.}

En aras de conocer esta perspectiva conservacionista aplicada al Parque Nacional Diría, hay que señalar que esta zona determinada representa y es un hito en materia de conservación donde dichosamente, las comunidades que se encuentran ubicadas en los alrededores han sido sujetos de educación ambiental, en particular la que ofrece la Universidad de Costa Rica en la modalidad de Trabajo Comunal Universitario o TCU.

\subsection{Parque Nacional Diriá}

El Parque Nacional Diría se encuentra geográficamente ubicado como parte territorial del Cantón de Nicoya y otra parte en el Cantón de Santa Cruz, ambos de la provincia de Guanacaste, al noroeste de Costa Rica y cuya cabecera cantonal es la Ciudad de Santa Cruz, también conocida como "la ciudad folclórica"; cuenta con una población de 60.4.495 habitantes según datos del año 2013 según el Instituto Nacional de Estádísticas y Censos (INEC). Corresponde a la localidad de Arado, distrito de Santa Cruz, cantón de Santa Cruz perteneciente a la provincia de Guanacaste. Según Leal (1998),

"la palabra «Diriá» de origen Chorotega tiene dos raíces: "Diri" que significa colina y "A" que significa pequeñita, en otras palabras significa Colinita. Esto fue, al parecer, lo que percibieron de esta ciudad originalmente: una pequeña colina entre los ríos Diría y En medio. Posteriormente la ciudad fue llamada Santa Cruz, al instalarse doña Bernabela Ramos en el valle del Diría y al colocar en el patio de su casa una gran cruz que sirvió también como lugar para realizar misas. El cantón de Santa Cruz fue oficialmente creado mediante la ley número 36, en su artículo número 9, el 7 de diciembre de 1848 y es el tercero de la provincia de Guanacaste. Para el año 1824, la población del cantón era de 2.502 habitantes, uno de los más poblados de toda la península guanacasteca. Para el año 1997, el cantón tenía una población de 41.112 habitantes y solo en el distrito primero (Santa Cruz) tenía 17.188 habitantes."

\section{(C) $(0 \bigcirc$}

La Revista Estudios es editada por la Universidad de Costa Rica y se distribuye bajo una Licencia Creative Commons Atribución-NoComercial-CompartirIgual 3.0 Costa Rica. Para más información envíe un mensaje a 
Especial: Humanismo e investigación: una actividad permanente en la Escuela de Estudios Generales

Este parque fue declarado zona protegida el 9 de julio de 1991 mediante el Decreto Ejecutivo 20517-MIRENEM. En el año 1993 se le asignó el nombre de Refugio Silvestre, pero en el 2004 obtuvo la categoría de Parque Nacional (Decreto Ejecutivo №32003-MINAE), pertenece al Área de Conservación Tempisque, cuenta con una extensión de 5.426 hectáreas en donde se protegen parte de las cuencas de los ríos conocidos como el Diriá, el "En medio" y el "Tigre" (http://areasyparques.com/areasprotegidas/parque-nacional-diria/). Su creación se

De hecho significativamente su creación se da principalmente para proteger las zonas de recarga de las cuencas de estos ríos, además para proteger la diversidad de fauna y flora, iniciativa llevada a cabo por los vecinos del cantón de Santa Cruz con el fin de asegurar el suministro de agua para el futuro (Sistema Nacional de Áreas de Conservación) ${ }^{1}$

- Con respecto a las zonas de vida, en la parte alta del Parque Nacional Diriá entre los $130 \mathrm{msnm}$ hasta los $983 \mathrm{msnm}$, subsisten los únicos remanentes de bosque tropical nuboso, que alguna vez cubrió las tierras altas de la Península de Nicoya; bosque húmedo transición a seco en la zona baja de la cuenca de los ríos Enmedio y Tigre y bosque muy húmedo premontano en las laderas de los ríos de Enmedio, Tigre, Diriá y cerro Vista del Mar (SINAC, 2013). La vegetación está conformada por remanentes de bosque primario, bosques de galería, tacotales (Terreno sin cultivar cubierto de maleza espesa) y bosques de crecimiento secundario. Es importante la conservación de los diferentes tipos de bosque para proteger poblaciones de venados, monos congo, osos colmeneros, saínos, diversidad de anfibios, variedad de insectos, reptiles, aves y

\footnotetext{
${ }^{1}$ El parque se caracteriza por presentar variedad de ecosistemas únicos en la Península de Nicoya. Véase al respecto: http://www.fundecodes.org/parque-nacional-diria/.

La Revista Estudios es editada por la Universidad de Costa Rica y se distribuye bajo una Licencia Creative Commons Atribución-NoComercial-Compartirlgual 3.0 Costa Rica. Para más información envíe un mensaje a revistaestudios.eeg@ucr.ac.cr.
} 
Especial: Humanismo e investigación: una actividad permanente en la Escuela de Estudios Generales

muchas otras especies que habitan los ecosistemas presentes en el parque (Boza y Cevo, 2001, p. 72).

Sus árboles son caducifolios, es decir pierden el follaje durante el verano, que se ven resecos, grises y sin vida, sin embargo debido a algunos aguaceros aislados y ocasionales se presenta el florecimiento de muchos de los árboles, cambiando el paisaje de aspecto seco en parches de gran vistosidad. Conforme avanza la época seca la cual se extiende aproximadamente del mes de diciembre al mes de abril, cuando llegan las lluvias (mayo a junio), el bosque se transforma en poco tiempo en un exuberante complejo de verdes follajes dando la sensación del retorno de la vida al bosque y produciéndose una segunda floración explosiva pletórica de vida que brinda alimento como néctar, frutos y semillas, todos suministros necesarios para la época reproductiva. Algunas especies características del bosque seco son el pochote (Bombacopsis quinata), Malacahuite (Chomelia spinosa), Guanacaste (Enterolobium cyclocarpum), cenízaro (Samanea saman), laurel (Cordia alliodora), roble sabana (Tabebuia rosea), balsa (Ochroma pyramidale) y Guácimo (Guazuma ulmifolia) entre otros. El bosque premontano muy húmedo presenta árboles de mediana altura y semiperennifolio, con copas anchas y redondas, se encuentran bejucos trepadores herbáceos y algunas epífitas (Meza, 2001. pp. 45-46).

La topografía es irregular. En el parque se encuentran dos de los tres principales picos montañosos de Santa Cruz que son el cerro Vista al Mar (983 msnm) y el cerro Retallano $(711 \mathrm{msnm})$ donde nacen los ríos Diriá y En medio. El cerro Vista al Mar es el segundo pico montañoso más alto de la Península de Nicoya, después del cerro Azul (1018 msnm).

\section{(c) (i) (9)}

La Revista Estudios es editada por la Universidad de Costa Rica y se distribuye bajo una Licencia Creative Commons Atribución-NoComercial-CompartirIgual 3.0 Costa Rica. Para más información envíe un mensaje a 
Especial: Humanismo e investigación: una actividad permanente en la Escuela de Estudios Generales

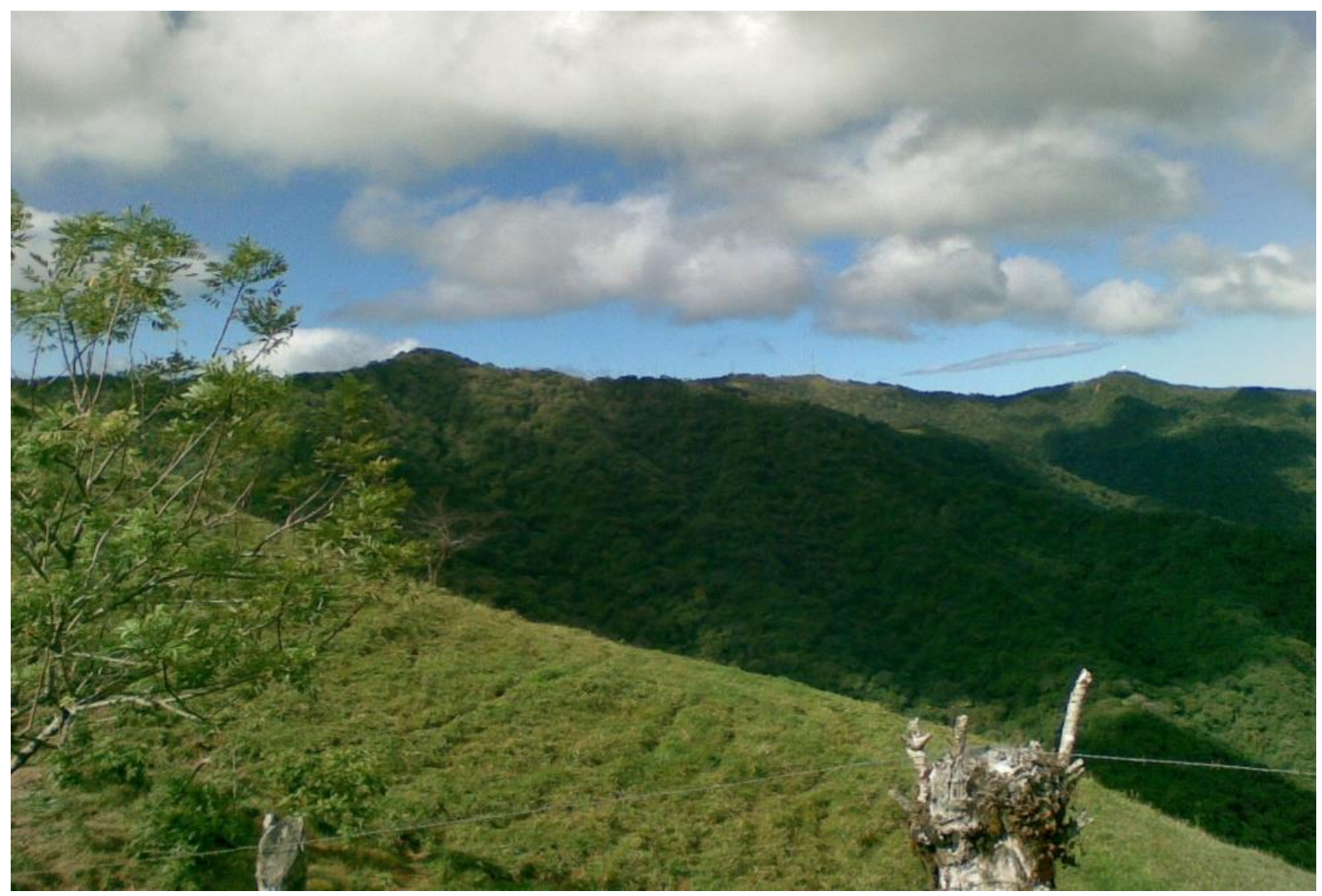

Vista panorámica del Parque Nacional Diría

Fuente: Ana L. Chavarría

24-04-2012

\subsection{Poblaciones cercanas al Parque Nacional Diriá}

Las comunidades que interactúan con el parque son principalmente: San Juan, El Arado, Vista Al Mar, Los Ángeles, La Esperanza, Colas de Gallo y Juan Díaz que pertenecen a los cantones de Santa Cruz y Nicoya, Guanacaste.

Fig.1: Muestra la ubicación de las comunidades cercanas al parque, la cuenca del río Enmedio y los principales ríos que abastecen de agua a la población de Santa Cruz.

\section{@(๑)@}

La Revista Estudios es editada por la Universidad de Costa Rica y se distribuye bajo una Licencia Creative Commons Atribución-NoComercial-CompartirIgual 3.0 Costa Rica. Para más información envíe un mensaje a revistaestudios.eeg@ucr.ac.cr. 
Especial: Humanismo e investigación: una actividad permanente en la Escuela de Estudios Generales

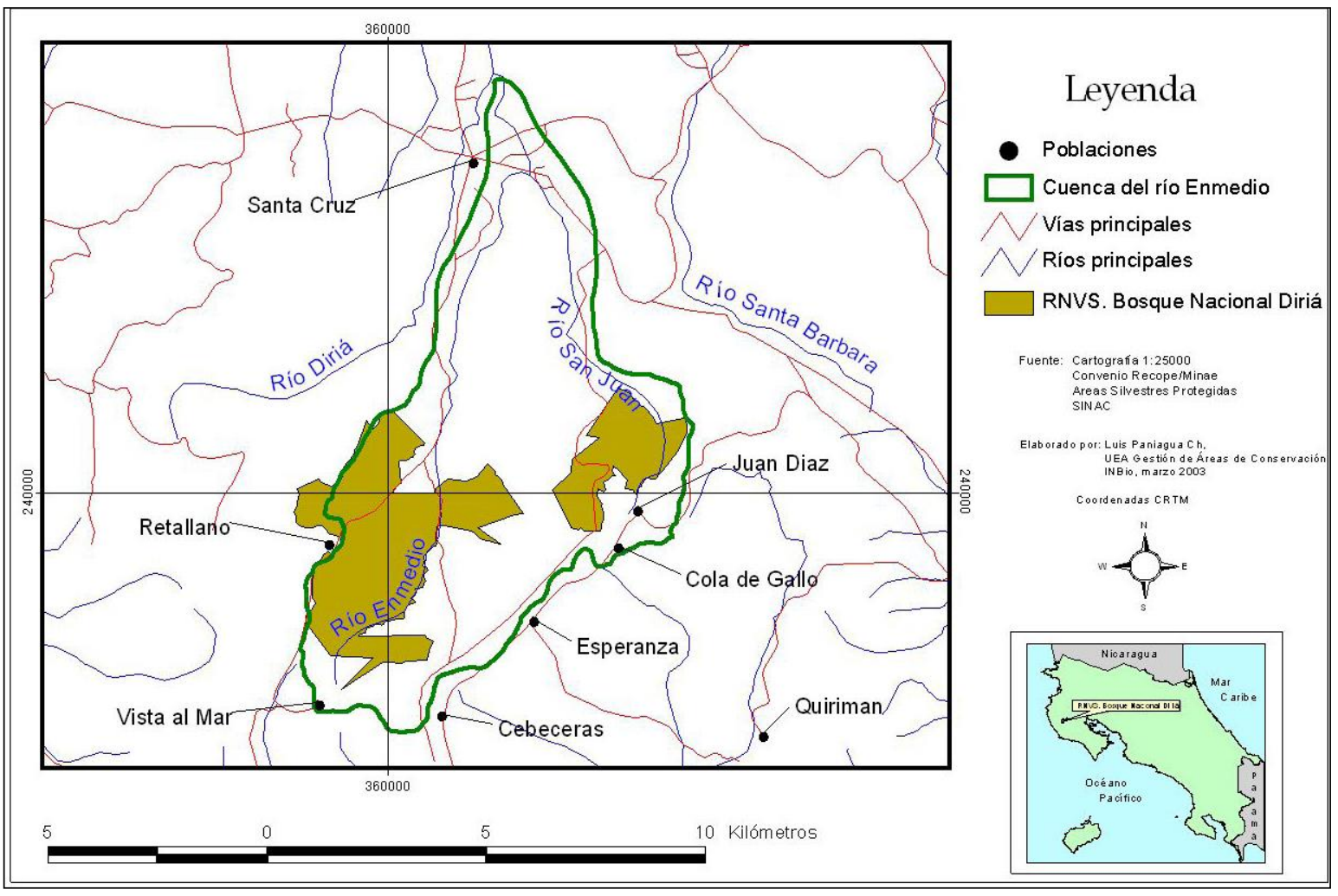

Fuente SINAC. 2002.

Estas comunidades en general son de baja condición socioeconómica y su principal actividad es la agricultura. Los habitantes de Colas de Gallo se dedican principalmente a las actividades agrícolas como son la siembra de frijoles, café y otros productos alimenticios de los cuales obtienen los recursos para el sustento diario de las familias. En cuanto a la educación primaria solo cuentan con una escuela uni- docente.

La comunidad de Juan Díaz se encuentra a $10 \mathrm{~km}$ del área administrativa del parque. Esta comunidad pertenece a Nicoya, al igual que Colas de Gallo cuya ubicación es a $8 \mathrm{~km}$ del parque. Los habitantes de Juan Díaz cuentan con Iglesia, una Escuela donde se imparten todos los niveles y un salón para actividades culturales y sociales. Al igual que Colas de Gallo sus pobladores se dedican

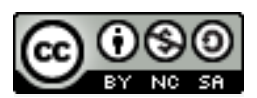

La Revista Estudios es editada por la Universidad de Costa Rica y se distribuye bajo una Licencia Creative Commons Atribución-NoComercial-CompartirIgual 3.0 Costa Rica. Para más información envíe un mensaje a revistaestudios.eeg@ucr.ac.cr. 
Especial: Humanismo e investigación: una actividad permanente en la Escuela de Estudios Generales

principalmente a las actividades agrícolas. También cuentan con una plaza de deportes y un negocio donde se adquieren víveres y artículos básicos.

La Esperanza es otra comunidad distante a $5.5 \mathrm{~km}$ del parque Diriá, con escuela y colegio. El colegio recientemente adquirió una planta física nueva algo de suma importancia ya que anteriormente recibían clases en el salón comunal. También existe un local que es utilizado como clínica visitada por un médico para atender las necesidades de los pobladores una vez al mes, fecha en la que acuden los niños, los jóvenes, los adultos y los ancianos que necesitados de atención médica o también para los pertinentes controles de rutina. Los habitantes se trasladan desde todos los puntos lejanos recorriendo en su mayoría a pie o a caballo las distancias establecidas pues la mayoría de los habitantes no poseen carro ni cuentan con servicio de transporte público interno.

Con respecto a la comunidad de los Ángeles que se encuentra a $9 \mathrm{~km}$ de la Casona, es pequeña al punto que la escuela cerrara las puertas ya que no cuenta con población estudiantil para los diferentes niveles escolares porque sus pobladores se han trasladado a las otras comunidades.

\section{(c) (i) (2)}

La Revista Estudios es editada por la Universidad de Costa Rica y se distribuye bajo una Licencia Creative Commons Atribución-NoComercial-CompartirIgual 3.0 Costa Rica. Para más información envíe un mensaje a 


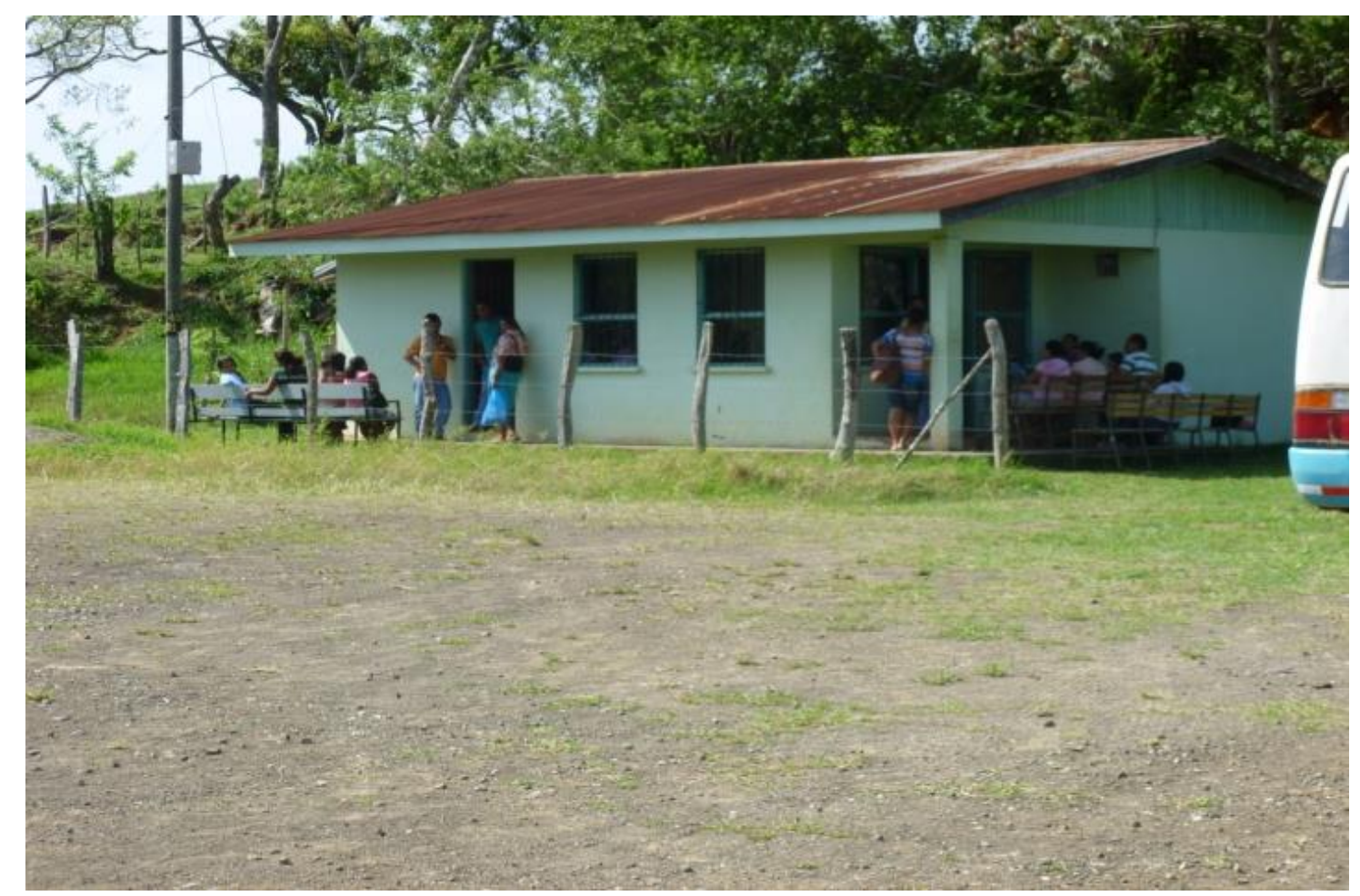

Centro de Salud de la Esperanza.

Fuente: Ana L. Chavarría 27-07-2014

Por último es importante hacer referencia a que en las diferentes regiones donde están localizadas las comunidades mencionadas anteriormente se cuenta con zonas dedicadas a la ganadería, alguna plantación forestal como melina, teca y pochote. En los sectores ubicados en altitudes superiores a 600 metros, existe una considerable actividad agrícola, siendo posible encontrar plantaciones de frijol, maíz, hortalizas, aguacate, café y cítricos, entre otras. Estas actividades son desarrolladas fundamentalmente por pequeños productores, las cuales pueden causar algunos problemas ambientales, como la misma deforestación y la erosión del suelo, entre otros. En los alrededores de esas comunidades quedan aún

\section{(@) $\odot \otimes \odot$}

La Revista Estudios es editada por la Universidad de Costa Rica y se distribuye bajo una Licencia Creative Commons Atribución-NoComercial-Compartirlgual 3.0 Costa Rica. Para más información envíe un mensaje a revistaestudios.eeg@ucr.ac.cr. 
Especial: Humanismo e investigación: una actividad permanente en la Escuela de Estudios Generales

algunos pequeños parches de bosques nativos, razón por la cual, fue necesario trabajar con los pobladores con el propósito de educar e informar sobre la necesidad de proteger los remanentes de bosque así, como también educar a los niños, jóvenes y a las comunidades en general sobre las consecuencias de extracción de animales y plantas del área del Parque Nacional Diriá (SINAC, 2013).

\section{IMPORTANCIA DEL PROYECTO DEL TCU-DIRIÁ.}

La Universidad de Costa Rica se ha preocupado por la proyección del quehacer institucional en la comunidad nacional mediante trabajos de campo actuando con docentes y estudiantes enfocados hacia las comunidades rurales y con el firme propósito institucional de comprender y buscar soluciones a los problemas locales de manera interdisciplinaria, para eso constan en muchas proyecciones específicas como en Diriá la participación de estudiantes educados en sendas carreras universitarias con la ejecución de proyectos institucionales avalados por la Vicerrectoría de Acción Social. Lo dicho, hace posible poner al servicio de las comunidades costarricenses la academia, la que a su vez, se fortalece de los conocimientos que aportan los sectores nacionales con los que ésta interactúa, es decir, se obtiene un mutuo enriquecimiento en el campo en que participan la universidad y la comunidad.

Más aún, el Trabajo Comunal Universitario (TCU), se estableció en el Estatuto Orgánico de la Universidad de Costa Rica (Normativa institucional que determina los Principios y Propósitos de la Universidad, su Estructura y Gobierno, así como el Régimen de Enseñanza, el Régimen Administrativo y las Disposiciones Generales del alma máter), como un requisito obligatorio de graduación para todos los estudiantes de la Universidad de Costa Rica, no como una práctica académica más, sino como una modalidad de Acción Social fundamental para la formación humanística de los futuros profesionales mediante

La Revista Estudios es editada por la Universidad de Costa Rica y se distribuye bajo una Licencia Creative Commons Atribución-NoComercial-CompartirIgual 3.0 Costa Rica. Para más información envíe un mensaje a 
Especial: Humanismo e investigación: una actividad permanente en la Escuela de Estudios Generales

proyectos dirigidos principalmente a los sectores en áreas de mayor vulnerabilidad social o para el desarrollo de estrategias que permitan llevar a cabo tareas concretas de apoyo y soluciones a los problemas comunales.

Dentro de la diversidad de proyectos de Trabajo Comunal en la Sede Rodrigo Facio, planteados por las diferentes unidades académicas, la Escuela de Biología desarrolló el proyecto inscrito TC-54: "Interpretación y educación ambiental en áreas silvestres protegidas de Costa Rica”. El propósito de este proyecto fue contribuir con la conservación y protección de las áreas silvestres asumiendo la formación y la capacitación de las comunidades cercanas a estas áreas. Es importante señalar que el proyecto se inicia en enero del 2006 y se concluye en el 2017, espacio temporal donde se realizaron proyectos en el Parque Nacional Barra Honda, Corredor Biológico Cerros de Jesús, Refugio Nacional de Vida Silvestre Ostional y para finalizar, la experiencia anterior se trasladada este al Parque Nacional Diriá donde se conocieron las necesidades propias del parque con las comunidades aledañas, todo con el fin de que estas comunidades asuman un sentido de pertenecía y con ello, lograr que los habitantes de estas comunidades se convirtieran en defensores y protectores de la diversidad de recursos que posee el parque.

El proyecto TC-54: "Interpretación y educación ambiental en áreas silvestres protegidas de Costa Rica” se inició en enero del 2006 en el Parque Nacional Diría cuyo coordinador fue Elmer Guillermo García, profesor de la Escuela de Biología y de la Escuela de Estudios Generales.

El TCU en mención, cuenta también con la colaboración por parte de los funcionarios del parque, ya que sin su aporte al TCU, como conocedores directos de las particularidades que la universidad desconoce, el proyecto no tendría los mismos éxitos alcanzados hasta el día de hoy.

\section{(c) (i) (2)}

La Revista Estudios es editada por la Universidad de Costa Rica y se distribuye bajo una Licencia Creative Commons Atribución-NoComercial-CompartirIgual 3.0 Costa Rica. Para más información envíe un mensaje a 
Especial: Humanismo e investigación: una actividad permanente en la Escuela de Estudios Generales

Las instalaciones con las que cuenta el parque son las usuales: dormitorios, cocina - comedor, servicio de agua potable y de electricidad; con capacidad para recibir a 25 personas, además cuenta con una zona de acampamiento abastecida con servicio de mesas, baños y sanitarios. Es necesario para el parque y para las comunidades cercanas, una mayor implementación en el desarrollo turístico con el fin de generar recursos pero teniendo en cuenta la necesidad de conservar los recursos del parque. Es fundamental que se trabaje en un desarrollo turístico donde las comunidades se beneficien mostrando sus costumbres, productos agrícolas y artesanías y se constituyan en defensores y protectores de los recursos del parque y que también genere recursos para sus habitante, en consecuencia la administración del parque hace esfuerzos para la construcción de un área para los visitantes, sin embargo, se necesita otro proyecto que permita a estos disfrutar de las bellezas naturales como las cataratas, observación de aves, vegetación exuberante y del caudal de los ríos de Enmedio y Diría. $^{2}$

En este sentido, se insiste en el hecho de que el parque cuenta con dormitorios para estudiantes e investigadores, agua potable, electricidad y un comedor con todo lo necesario, sanitarios y áreas para acampar. También se pueden realizar caminatas por los senderos Espavel y El Venado donde se puede observar fauna, flora, disfrutar de la belleza de los ríos Diría, el de En medio, quebradas, cataratas Brasil y Santa Fe, entre muchas razones por las que es de suma importancia proteger y conservar toda la riqueza para disfrute de la población costarricense y principalmente para los ciudadanos guanacastecos.

\footnotetext{
${ }^{2}$ Se están haciendo diagnósticos de las posibles estrategias de desarrollo turístico viable para esta zona por parte de los estudiantes de la carrera de Turismo, proyectos que están

(c) (i) (8)

La Revista Estudios es editada por la Universidad de Costa Rica y se distribuye bajo una Licencia Creative Commons Atribución-NoComercial-CompartirIgual 3.0 Costa Rica. Para más información envíe un mensaje a revistaestudios.eeg@ucr.ac.cr.
} 
Especial: Humanismo e investigación: una actividad permanente en la Escuela de Estudios Generales

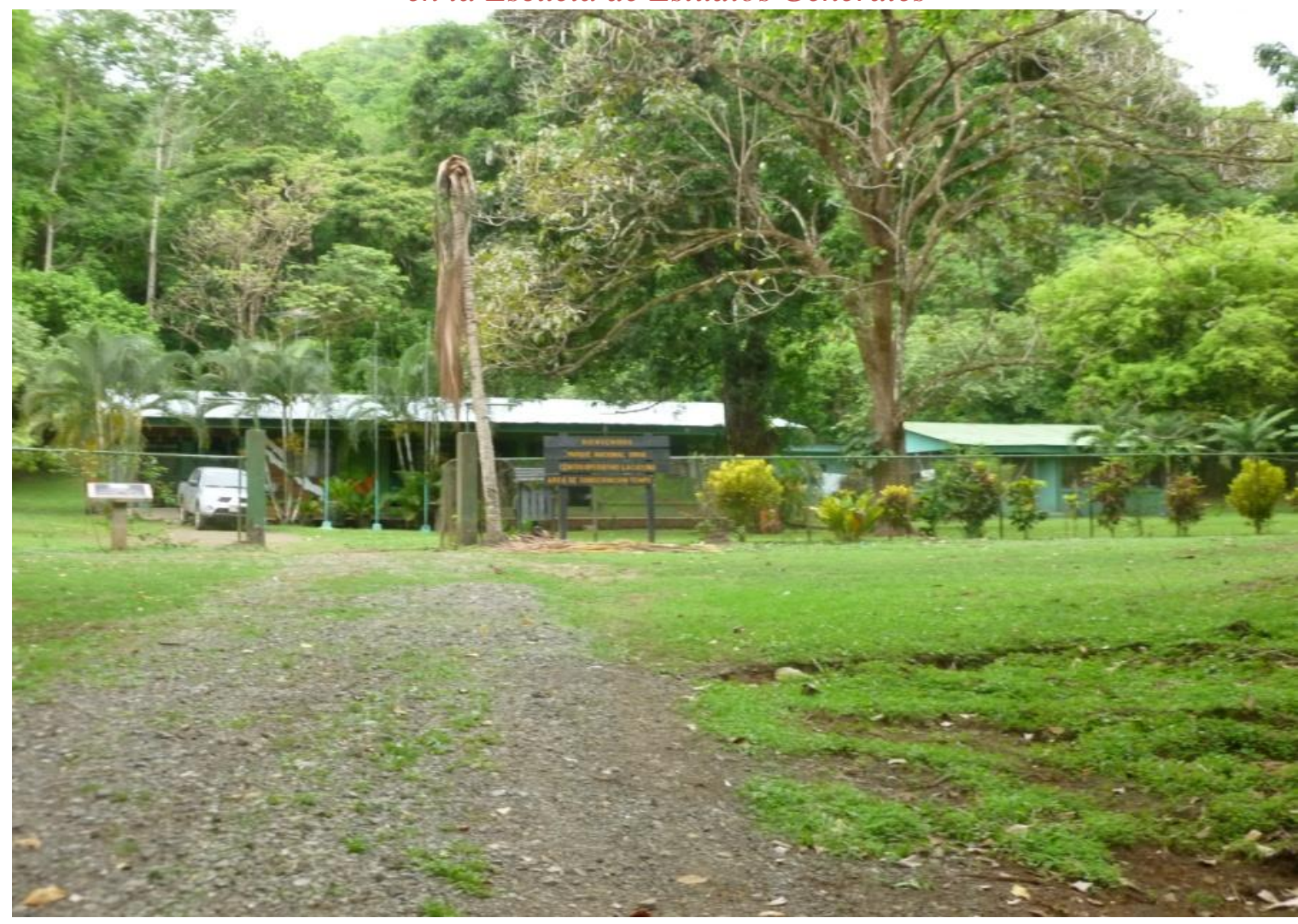

Oficinas Administrativas

Entrada principal al Parque Nacional Diría

Fuente: Ana L. Chavarría

5. Aportes del Trabajo Comunal Universitario proyecto TC-54: "Interpretación y educación ambiental en áreas silvestres protegidas de Costa Rica")

En visitas realizadas durante 7 años (2009-2017) al Parque Nacional Diría quiero resaltar las labores realizadas por los estudiantes de las diferentes carreras, que dieron su aporte con el fin de cubrir algunas necesidades del parque y de las comunidades que colindan con dicho parque. También es importante mencionar que los estudiantes tienen la oportunidad de poner en práctica sus

\section{(a) $(\Theta \odot$}

La Revista Estudios es editada por la Universidad de Costa Rica y se distribuye bajo una Licencia Creative Commons Atribución-NoComercial-CompartirIgual 3.0 Costa Rica. Para más información envíe un mensaje a revistaestudios.eeg@ucr.ac.cr. 
Especial: Humanismo e investigación: una actividad permanente en la Escuela de Estudios Generales

respectivas carreras y a la vez compartir con las comunidades en cuanto a costumbres, historias de sus pueblos y actividades que les permiten un aprendizaje diferente al brindado en las aulas de la universidad lo que contribuye a engrandecer su formación.

\subsection{Primer aporte.}

Los estudiantes de la carrera de Biología, han realizado un muestreo de especies tanto de flora como de fauna con el fin de establecer un registro de la biodiversidad que habita en el Parque Nacional Diriá. Es importante identificar las diferentes especies para fundamentar la necesidad de proteger la riqueza de todos los ecosistemas existentes y el manejo del mismo. Para mencionar algunos trabajos realizados durante el desarrollo del proyecto, los estudiantes que participaron con la ayuda que brindaron especialistas en este campo, se han logrado identificar 22 especies de hongos, 30 especies de briófitos, 17 especies de arañas, 13 especies de reptiles, 43 especies de murciélagos y 15 especies de mamíferos, entre otras. Todos estos ejemplos constituyen una muestra del trabajo realizado.

Los proyectos de identificación de especies les permiten a los estudiantes de biología aplicar sus conocimientos académicos adquiridos durante la carrera y haciendo materialmente visible un importante aporte científico al país.

\subsection{Segundo aporte.}

Otra actividad realizada fue la capacitación de los agricultores en el tratamiento de los desechos orgánicos utilizando para estos fines la lombricultura con la lombriz californiana. Se les brindó información precisa para el manejo adecuado de la técnica y así también resolver el problema de la inexistencia del servicio de recolección de basura Municipal.

\section{(c) (i) (2)}

La Revista Estudios es editada por la Universidad de Costa Rica y se distribuye bajo una Licencia Creative Commons Atribución-NoComercial-CompartirIgual 3.0 Costa Rica. Para más información envíe un mensaje a 
Especial: Humanismo e investigación: una actividad permanente en la Escuela de Estudios Generales

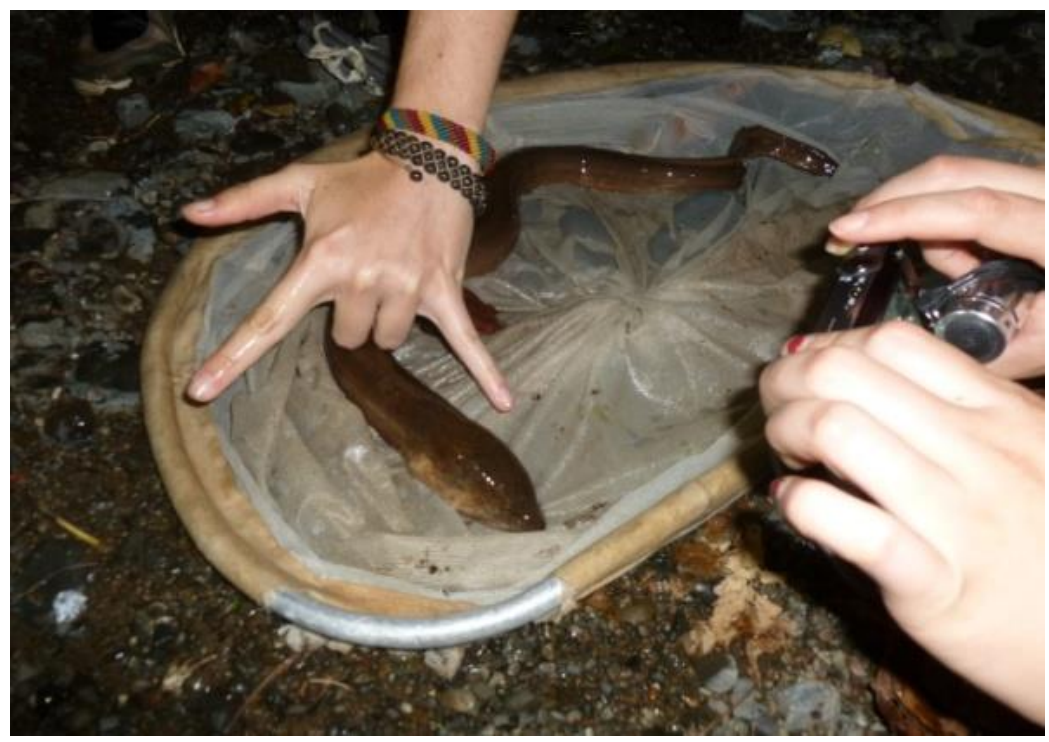

Estudiantes muestreando especies que se encuentran en el Río de En medio Fuente: Ana L. Chavarría (18-12-2007)

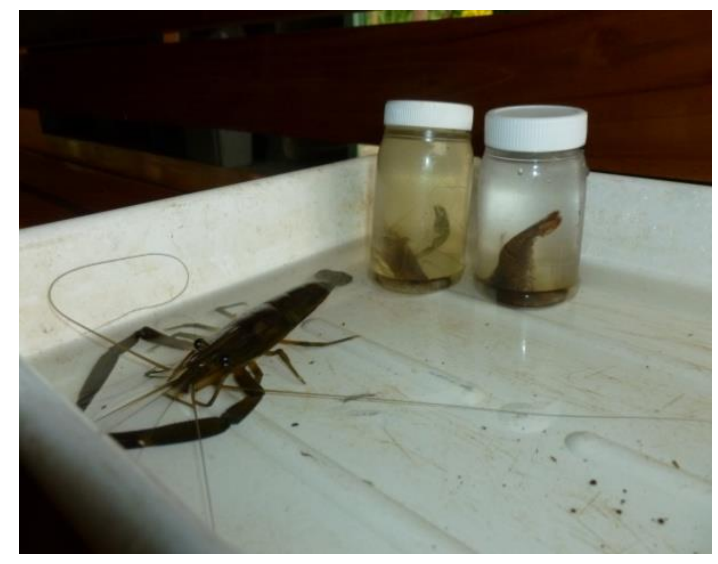

Fuente: Ana L. Chavarría (27-12-2007)

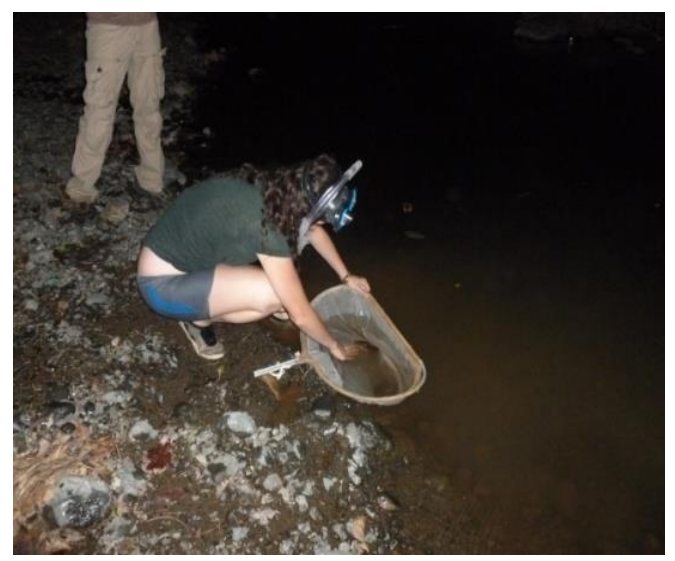

Fuente: Ana L. Chavarría 27-12-207

\section{(ब) $(\Theta 0$}

La Revista Estudios es editada por la Universidad de Costa Rica y se distribuye bajo una Licencia Creative Commons Atribución-NoComercial-CompartirIgual 3.0 Costa Rica. Para más información envíe un mensaje a revistaestudios.eeg@ucr.ac.cr. 
Especial: Humanismo e investigación: una actividad permanente en la Escuela de Estudios Generales

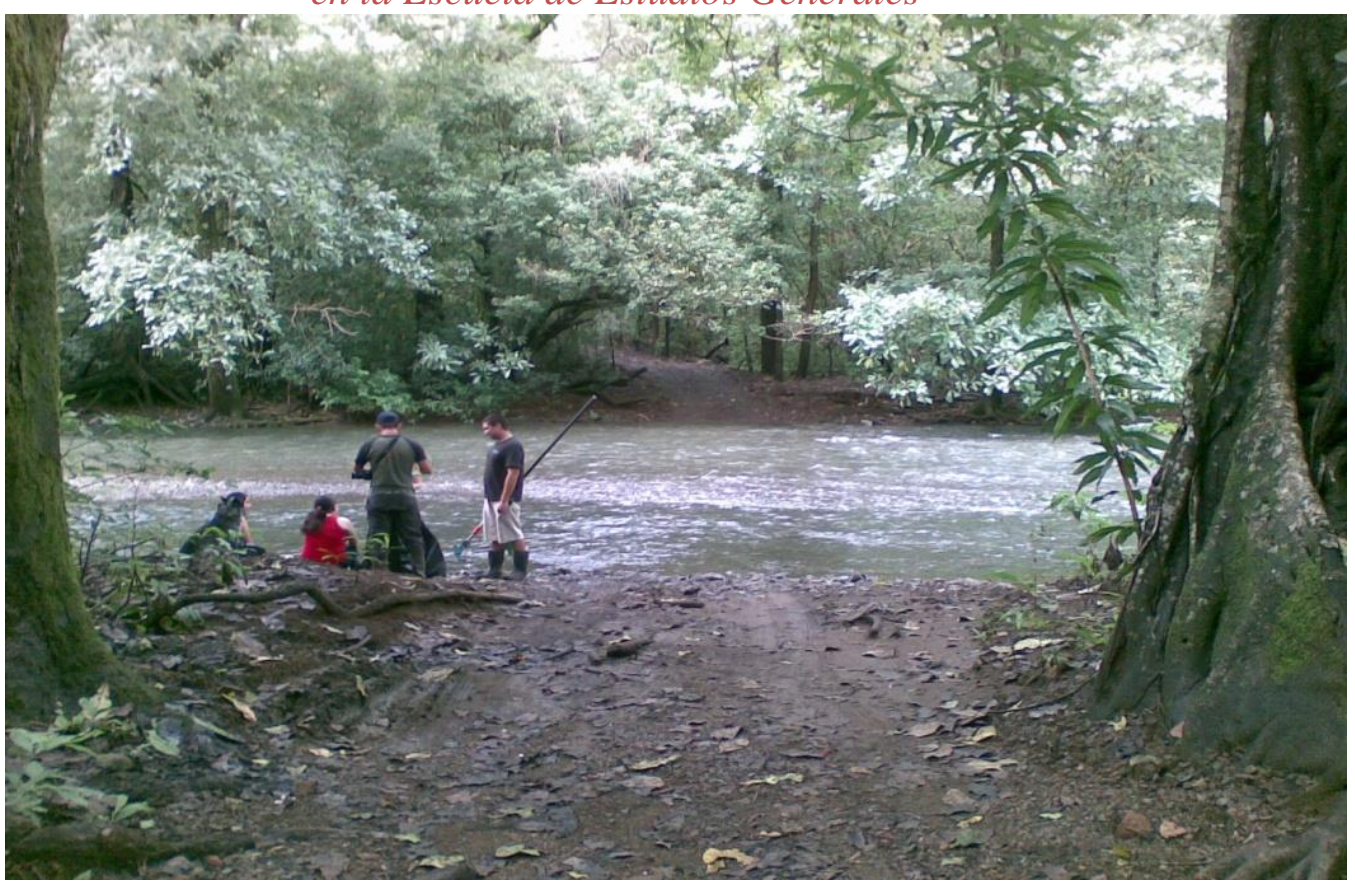

Fuente: Ana Chavarría 18-12-2013

\subsection{Tercer aporte.}

Corresponde al aporte de los estudiantes de la carrera de Educación quienes realizaron variedad de actividades en las Escuelas de las comunidades arriba mencionadas dando charlas informativas, y confeccionando títeres con los niños relacionados con los temas ambientales. Además, realizan talleres y festivales con el fin de acercar a los pobladores de las diferentes comunidades. Todas las actividades hechas por los estudiantes tenían como propósito informar y educar a los niños y niñas, hacerles ver la importancia de la conservación de los recursos del parque y cómo evitar la contaminación de los ríos, la extracción de fauna y flora, el uso adecuado de sustancias químicas en los cultivos, la necesidad de implementar prácticas de reciclaje para los desechos sólidos

Por último en las comunidades también se trabajó con la población adulta, tanto con las mujeres de casa como con los agricultores. Con las amas de casa

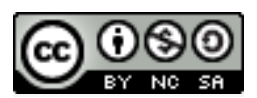

La Revista Estudios es editada por la Universidad de Costa Rica y se distribuye bajo una Licencia Creative Commons Atribución-NoComercial-CompartirIgual 3.0 Costa Rica. Para más información envíe un mensaje a revistaestudios.eeg@ucr.ac.cr. 
Especial: Humanismo e investigación: una actividad permanente en la Escuela de Estudios Generales

los estudiantes de Microbiología realizando charlas sobre prácticas para el mejoramiento de la salud y el de la higiene personal. Por eso se atiende la verificación de la calidad hídrica con la recolección de muestras de agua de los ríos de donde se toma el agua que se consume en las viviendas y se utilizan los recursos de los laboratorios universitarios para su estudio. Luego, en visitas posteriores son entregados los resultados con el propósito de que se tomen las medidas correctivas que traigan mayor cuidado de las aguas.

\subsection{Cuarto aporte.}

Consta arriba que los pobladores se dedican principalmente a la producción agrícola y a la ganadería vacuna. Por eso, con los agricultores los estudiantes de Agronomía se dieron a la tarea de brindarles información sobre los problemas de contaminación de los suelos y las aguas por el uso de agroquímicos; entonces se les enseña el manejo adecuado de los desechos con la ya mencionada técnica de la lombricultura y el adecuado manejo de agroquímicos de la manera más racional y adecuada. ${ }^{3}$

\subsection{Quinto aporte.}

Por otra parte la acción de los estudiantes de la Escuela de Artes, se ha enfocado en el diseño y ejecución de murales para las escuelas locales, centros comunales y también en la casona del parque con motivos que reflejan las costumbres populares, algunas de las tradiciones culturales de la zona, y evidenciar la existencia de la fauna y la flora característica; embellecen los espacios e informan sobre aspectos culturales y ambientales (Fotos 1,2).

\footnotetext{
${ }^{3}$ Es importante destacar que las relaciones que se establecen entre los habitantes de las comunidades y los estudiantes en un aprendizaje mutuo ya que les brindan hospedaje mientras realizan su proyecto.
}

\section{(c) (i) (2)}

La Revista Estudios es editada por la Universidad de Costa Rica y se distribuye bajo una Licencia Creative Commons Atribución-NoComercial-CompartirIgual 3.0 Costa Rica. Para más información envíe un mensaje a 
Especial: Humanismo e investigación: una actividad permanente

en la Escuela de Estudios Generales

En este sentido es importante mencionar el trabajo realizado durante el segundo semestre del 2011 en el Salón Comunal de La Esperanza por la creación y el área del mural (Fotos 3,4), la belleza, diseño artístico y la combinación de cultura y ambiente que enriquece el paisaje de la comunidad donde estudiantes y pobladores disfrutan de la magnífica obra de arte realizado por los estudiantes de arte y de las otras áreas que conforman el grupo del TCU. Los murales de los centros educativos son realizados como ya se dijo por los estudiantes encargados pero también consta la participación de los niños y niñas, lo que genera una apropiación del mismo y un cuidado por parte de los centros educativos hacia estas obras consideras por ellos muy importantes (Fig.3). También se ha realizado un mural en la Casona del parque, tomando en cuenta sus alrededores y dando una visión de la fauna y flora que lo rodea (Foto 5).

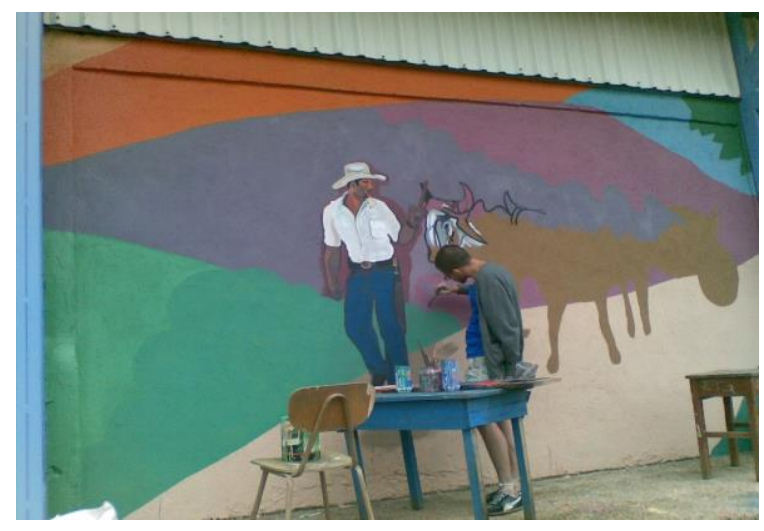

Fuente: Ana L. Chavarría 20-12-2010
Mural en el Colegio de la Comunidad de La Esperanza

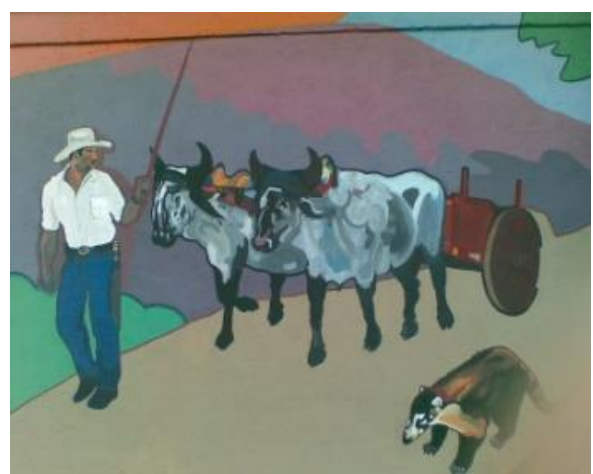

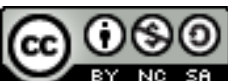

La Revista Estudios es editada por la Universidad de Costa Rica y se distribuye bajo una Licencia Creative Commons Atribución-NoComercial-CompartirIgual 3.0 Costa Rica. Para más información envíe un mensaje a revistaestudios.eeg@ucr.ac.cr. 
Especial: Humanismo e investigación: una actividad permanente en la Escuela de Estudios Generales

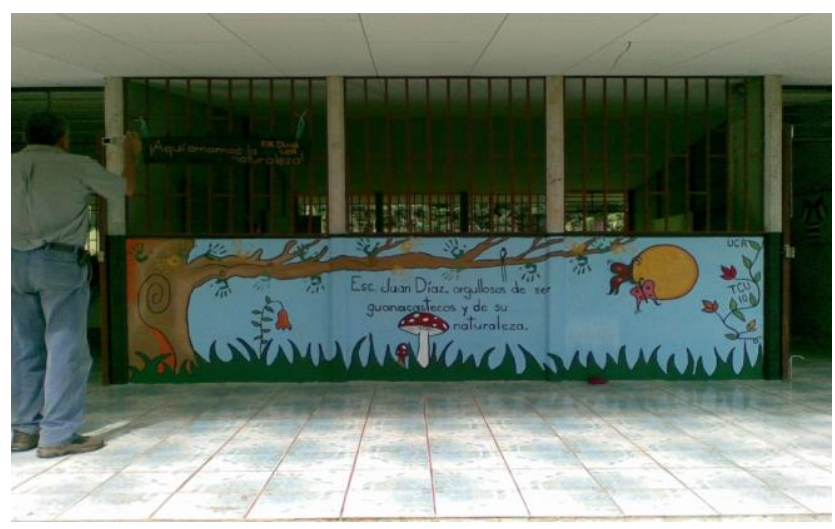

Mural en la Escuela de la Comunidad de Juan Díaz Fuente. Ana L. Chavarría 18-122008

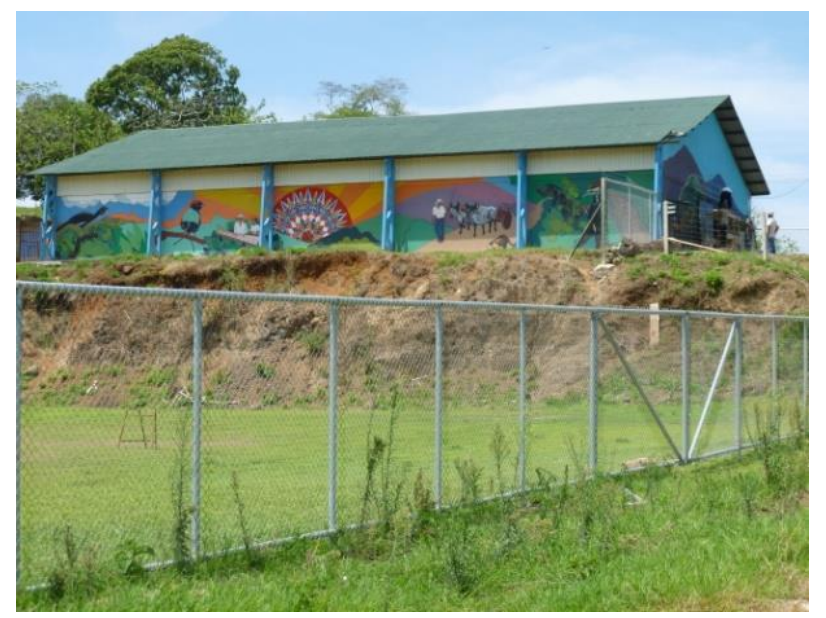

Mural realizado en el Salón

Comunal de Juan Díaz

Fuente: Ana L. Chavarría. 12-

12-2009

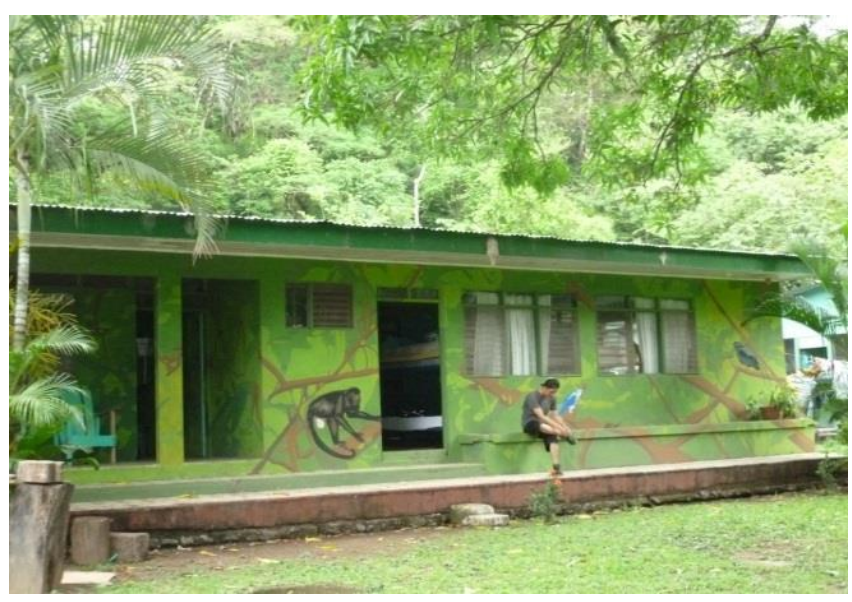

Mural realizado en la Casona de el Parque Nacional Diría

Fuente: Ana L. Chavarría 17-12-2009

\section{(C) $\odot \Theta(0$}

La Revista Estudios es editada por la Universidad de Costa Rica y se distribuye bajo una Licencia Creative Commons Atribución-NoComercial-CompartirIgual 3.0 Costa Rica. Para más información envíe un mensaje a revistaestudios.eeg@ucr.ac.cr. 
Especial: Humanismo e investigación: una actividad permanente en la Escuela de Estudios Generales

\subsection{Sexto aporte}

Los educandos de la carrera de informática crearon una página en la web para consulta del público con las características del parque, su geología, su historia y la ruta para la visita, así como los recursos con que cuentan los visitantes en cuanto a instalaciones y senderos. Los estudiantes de Geología se ocuparon de una de las necesidades del área del parque que es la construcción de los mapas sobre el uso del suelo y el establecimiento de los límites del parque, aspecto importante que constituía un vacio para la administración del parque.

\section{Conclusiones}

Para el proyecto T-54: "Interpretación y educación ambiental en áreas silvestres protegidas de Costa Rica" ha sido muy satisfactorio el trabajo realizado y que constituye una motivación para seguir realizando este tipo de actividades humanísticas donde los estudiantes de la Universidad de Costa Rica ponen muy en alto a la institución que representan y donde las comunidades y funcionarios del parque manifiestan su agradecimiento por brindarles información y trabajo que benefician tanto al parque como a las comunidades.

La Universidad de Costa Rica brinda una proyección positiva dentro de uno de los ejes de la institución como lo es la Acción Social. Todo este trabajo no sería posible sin el compromiso y apoyo brindado por la administración y los funcionarios del Parque Nacional Diría.

Son muchos los proyectos desarrollados por los estudiantes de la Universidad de Costa Rica en sus trabajos comunales, la diversidad de carreras como Medicina, Ingeniería Industrial, Trabajo Social, Educación, Microbiología, Artes Plásticas y otras, los cuales han aportado conocimientos profesionales 
Especial: Humanismo e investigación: una actividad permanente en la Escuela de Estudios Generales

adquiridos en sus procesos de aprendizaje a cada una de las comunidades y al Parque Nacional Diría. Dichos estudiantes han recibido a lo largo de su experiencia el agradecimiento por su desinteresada labor.

Estar en contacto de forma directa con los pobladores de las comunidades, con los niños y niñas de las escuelas, los estudiantes del Colegio La Esperanza, en actividades que permiten conocer las necesidades y costumbres de los pobladores y al mismo tiempo buscar en lo posible soluciones con los conocimientos que han adquirido en los años de estudio desde sus respectivas carreras, es un reto que permite en conjunto con la participación de los líderes comunales, la posibilidad de soluciones integrales. La acción social desarrollada por la Universidad de Costa Rica en las comunidades antes mencionadas y en el Parque Nacional Diría por parte del TCU, ha sido fundamental no solo para mejorar los cambios de conducta y conocimiento con respecto a las áreas protegidas referentes a su protección, valoración y cuido por parte de las comunidades sino también que las actividades y desarrollo del proyecto contribuya a mejorar la calidad de vida de los habitantes de las comunidades.

Se pretende que el parque y las comunidades en el futuro se beneficien de un desarrollo sustentable, donde las actividades realizadas permitan la protección de los recursos, pero que al mismo tiempo las comunidades se desarrollen y obtengan recursos que les permitan una armonía con el Parque Nacional Diría. Se debe enfatizar en la protección de la biodiversidad y de los recursos en general, sin olvidar la importancia de los aspectos sociales, económicos culturales y políticos de las comunidades que rodean el parque y que constituye un papel fundamental dentro de los objetivos del proyecto desarrollado por los estudiantes de trabajo comunal universitario.

\section{(a) $(\triangle \odot$}

La Revista Estudios es editada por la Universidad de Costa Rica y se distribuye bajo una Licencia Creative Commons Atribución-NoComercial-CompartirIgual 3.0 Costa Rica. Para más información envíe un mensaje a revistaestudios.eeg@ucr.ac.cr. 
Especial: Humanismo e investigación: una actividad permanente en la Escuela de Estudios Generales

Se les agrádese a todos los estudiantes que han participado en el proyecto de TCU desde enero de 2006. - 2017

\section{Referencias Bibliográficas}

Aéreas de Parques Nacionales y Zonas Protegidas. http://areasyparques.com/areasprotegidas/parque-nacional-diria// (fecha de visita 24 de julio 2017).

Blanco, O. y A. Mata. (1994) La Cuenca del Golfo de Nicoya: un reto al desarrollo sostenible. 1. Ed. San José, Costa Rica: Editorial de la Universidad de Costa Rica.

Ecosistemas de Costa Rica. Blog de la biodiversidad de Costa Rica http://ecosistemasdecostarica.blogspot.com/2012/08/parque-nacional-diria.html (fecha de visita 24 julio 2017)

Forgen, M. (1997) Vida silvestre de los parques y reservas de Costa Rica. San José, Costa Rica: Editorial Heliconia.

Instituto Nacional de Biodiversidad de Costa Rica (2017). Recuperado: 23 julio 2017. http://www.inbio.ac.cr/17-efemerides.html

Instituto Nacional de Biodiversidad (2012) Instituto Nacional de Biodiversidad (en línea). www.inbio.ac.cr

Leal, E. (1998). Santa Cruz: El paraje del Diría. E. Leal A. San José Costa Rica http://www.inbio.ac.cr/es/estudios/PDF/Informe EvalEcologicaDiria.pdf

Mesa Ocampo, Tobías. (2001) Geografía de Costa Rica. Cartago: Editorial Tecnológica de Costa Rica.

Morera, C. (2016) "Evaluación de la cobertura boscosa en Costa Rica: un análisis a nivel de las áreas de conservación en el año 2000". Revista Geográfica de América Central N`56 pp163- 181.

https://www.researchgate.net/publication/303484343 EVALUACION DE LA COB ERTURA BOSCOSA EN COSTA RICA UN ANALISIS A NIVEL DE AREAS DE CONSERVACION EN EL ANO 2000 (fecha de visita 24 julio del 2017)

La Revista Estudios es editada por la Universidad de Costa Rica y se distribuye bajo una Licencia Creative Commons Atribución-NoComercial-CompartirIgual 3.0 Costa Rica. Para más información envíe un mensaje a revistaestudios.eeg@ucr.ac.cr. 
ISSN 1659-3316

Especial: Humanismo e investigación: una actividad permanente en la Escuela de Estudios Generales

Obando, V. (2002) Biodiversidad en Costa Rica: Estado del conocimiento y gestión. Primera Edición. Santo Domingo de Heredia, Costa Rica: Instituto Nacional de Biodiversidad.

SINAC. (1964) Parque Nacional Diría. Slud, P. The birds of Costa Rica: distribution and ecology. Bulletin of the American Museum of Natural En:http://www.sinac.go.cr/act_bosquediria_general.php History, 128.

Stiles; A. S. (2007). Guía de Aves de Costa Rica. Cuarta edición. Costa Rica: Instituto Nacional De Biodiversidad (INBio). 